Research Paper

\title{
Cysteine-rich 61 (Cyr61) upregulated in pulmonary arterial hypertension promotes the proliferation of pulmonary artery smooth muscle cells
}

\author{
Lan Gao ${ }^{1,2,}$, , Yong Fan ${ }^{1}{ }^{\dagger}$, Yanjie Hao1, Ping Yuan ${ }^{3}$, Dong Liu ${ }^{3}$, Zhicheng Jing 3,4 , Zhuoli Zhang ${ }^{1, \bowtie}$ \\ 1. Department of Rheumatology and Clinical Immunology, Peking University First Hospital, Beijing, China; \\ 2. Department of Rheumatology, Beijing Shijitan Hospital, Capital Medical University, Beiijing, China; \\ 3. Department of Cardio-Pulmonary Circulation, Shanghai Pulmonary Hospital, Tongji University, School of Medicine, Shanghai, China; \\ 4. State Key Laboratory of Cardiovascular Disease, Fu Wai Hospital, Peking Union Medical College and Chinese Academy Medical Science, Beijing, China; \\ † Drs. Lan Gao and Yong Fan contributed equally to this study. \\ $\square$ Corresponding author: Prof. Dr., Zhang Zhuoli, MD, PhD, Department of Rheumatology and Clinical Immunology, Peking University First Hospital, No.8, \\ Xishiku Street, West District, Beijing 100034, China. Email: zhuoli.zhang@126.com; Phone/Fax: +86-10-83572064
}

(c) Ivyspring International Publisher. This is an open access article distributed under the terms of the Creative Commons Attribution (CC BY-NC) license (https://creativecommons.org/licenses/by-nc/4.0/). See http://ivyspring.com/terms for full terms and conditions.

Received: 2017.01.21; Accepted: 2017.05.22; Published: 2017.07.19

\begin{abstract}
Background: We aimed to evaluate the expression of cysteine rich 61 (Cyr61) in patients with pulmonary arterial hypertension (PAH) as well as monocrotaline (MCT) induced PAH rat, and further investigate the effects and potential mechanisms of Cyr61 on the proliferation of pulmonary arterial smooth muscle cells (PASMCs).

Methods and Results: Plasma samples were collected from 20 patients with idiopathic PAH, 20 connective tissue disease (CTD) associated PAH, 29 age-, gender- and disease matched CTD without PAH patients, and 28 healthy controls. ELISA was used to detect the level of Cyr61 in plasma. MCT-induced PAH (MCT-PAH) rat model was established by a single subcutaneous injection of MCT $(60 \mathrm{mg} \mathrm{kg}-1)$. Lung tissues and pulmonary arteries of rats were collected, while the PASMCs were dissected and cultivated for in vitro experiments. Expression of Cyr61 in the lung tissues, pulmonary arteries and PASMCs were tested by immunohistochemical staining, western blot and quantitative real-time polymerase chain reaction. PASMCs from PAH rats were stimulated by exogenous recombinant Cyr61 protein and knocked down by small interfering RNA. Cell Counting Kit-8 assay was used to identify cell proliferation and the expression of p-AKT and AKT were analysed by western blot. The results showed plasma level of Cyr61 in PAH patients, especially CTD-PAH patients, were significant higher than that of CTD without PAH patients and healthy controls. Compared with wild rats, Cyr6I was overexpressed in the lung tissue, pulmonary arterial and PASMCs in PAH rats. Exogenous recombinant Cyr61 protein promoted the proliferation of PASMCs in a dose-dependent manner. While the expression of Cyr61 in PASMCs was inhibited by specific siRNA, cell proliferation was restrained and the expression of p-AKT declined.
\end{abstract}

Conclusion: Plasma Cyr61 concentration in PAH patients was highly increased. Cyr61 could promote PASMCs proliferation via AKT pathway, indicating that Cyr61 may play a role in the pathogenesis of $\mathrm{PAH}$.

Key words: Cysteine rich 61, pulmonary arterial hypertension, pulmonary arterial smooth muscle cells, proliferation

\section{Introduction}

Pulmonary arterial hypertension (PAH) is a sustained increase in pulmonary arterial pressure devastating disease characterized by a progressive (PAP), leading to right heart failure and death. It 
belongs to the first group of pulmonary hypertension $(\mathrm{PH})$ according to the classification of $\mathrm{PH}$ in the guidelines and hemodynamically characterized by the presence of pre-capillary $\mathrm{PH}$ including an end-expiratory pulmonary artery wedge pressure (PAWP) $\leq 15 \mathrm{~mm} \mathrm{Hg}$ and a pulmonary vascular resistance $>3$ Wood Units [1]. Based on the worldwide data, idiopathic PAH (IPAH) and connective tissue diseases associated pulmonary arterial hypertension (CTD-PAH) are the most common types of PAH. Despite the modern management era of $\mathrm{PAH}$, the functional limitation and survival of these patients remain unsatisfactory, especially CTD-PAH patients [2].

Actually, the fundamental pathogenesis of $\mathrm{PAH}$ is complex and remains poorly understood. Pulmonary vascular remodeling is a pathognomonic feature of $\mathrm{PAH}$, characterized by disordered proliferation and migration of pulmonary arterial smooth muscle cells (PASMCs) [3, 4]. In recent decades, an expanding body of knowledge indicated that inflammation and immunologic dysfunction underlie the development of PAH [4-6]. Pulmonary vascular lesions occurring in patients with $\mathrm{PAH}$ as well as in animal models are characterized by varying degrees of perivascular inflammatory cells infltrates, comprising $\mathrm{T}$ - and B-lymphocytes, macrophages, dendritic cells, and mast cells.

Cysteine rich 61 (Cyr61) is a secreted extracellular matrix-associated signaling molecule, which is $42 \mathrm{kd}$ and encoded by a growth factor-inducible immediate-early gene. It is produced and secreted by several cell types including endothelial cells, fibroblasts and smooth muscle cells. Cyr61 is remarkably versatile and can promote cell migration, adhesion, proliferation and angiogenesis under pathological conditions. Cyr61 also plays essential roles in normal biological events including embryonic development, angiopoiesis and wound healing [7-9]. Furthermore, Cyr61 has emerged as an important partner that controls the migration of leukocytes, the production of cytokines and the inflammatory process [10]. Considering the effect of Cyr61 on promoting proliferation and inflammation, we hypothesized that Cyr61 could participate in the pathogenesis of PAH. Very recently, Seon et al. [11] have revealed that Cyr61 was over-expressed in the pulmonary vessels and lung parenchyma in hypoxia-induced PAH mice. Additionally, Cyr61 could suppress pulmonary vascular smooth muscle contraction in response to hypoxia. However, the role of Cyr61 in monocrotaline-induced PAH has not been explored yet, and more importantly, we don't know the expression of Cyr61 in PAH patients and its role on PASMCs proliferation. Therefore, the objectives of this study were to evaluate the expression of Cyr61 in $\mathrm{PAH}$ patients and MCT induced PAH rats, and explore its potential role on PASMCs proliferation.

\section{Methods}

\section{Patients and specimens}

Between Jan 2009 and Aug 2012, twenty CTD-PAH patients were referred to Peking University First Hospital Rheumatology and Clinical Immunology department for PAH management (3 systemic sclerosis, 10 systemic lupus erythematosus, 3 mixed connective tissue disease, 3 Sjogren's syndrome, and 1 Behcet's disease), they were not previously treated with any $\mathrm{PAH}$-specific therapies. 29 age-, sex- and disease-matched CTD patients without PAH (3 systemic sclerosis, 17 systemic lupus erythematosus, 5 primary Sjogren's syndrome, and 4 Behcet's disease), 28 healthy controls from the same institute, and 20 age- and sex-matched idiopathic pulmonary arterial hypertension (IPAH) patients from the department of Cardio-Pulmonary Circulation, Shanghai Pulmonary Hospital were enrolled as different control groups. The diagnosis of PAH was established by right-heart catheterization and defined by a mean pulmonary artery pressure $\geq$ $25 \mathrm{~mm} \mathrm{Hg}$ at rest, an end-expiratory pulmonary artery wedge pressure (PAWP) $\leq 15 \mathrm{~mm} \mathrm{Hg}$ and a pulmonary vascular resistance $>3$ Wood units [12]. Plasma was obtained from each of the above patients and healthy individuals.

This study was conducted according to the principles of the Declaration of Helsinki, and was approved by Ethics Committee of Peking University First Hospital and Shanghai Pulmonary Hospital. Written informed consent was obtained from all patients when they were recruited to the study.

\section{Measurement of Cyr6 1 by ELISA}

Concentration of Cyr61 in plasma was measured quantitatively by enzymelinked immunosorbent assay kit (R\&D, USA). Briefly, microtiter plates were coated with capture antibody and stored overnight at room temperature. After washing with wash buffer for three times and educating with reagent diluent for 1 hour, standard substance and the plasma samples were added into the plates and incubated at room temperature for 2 hours. And then the plates were coated with detection antibody for additional 2 hours. After washing, streptavidin-conjugated horseradish peroxidase was used for color development. The absorbance of wells was detected by Microplate System (BioTek, USA) at 450nm. Finally, the concentration of Cyr61 was evaluated using ELISA Calc software. 


\section{Protocols for animal experiment}

Male Sprague-Dawley rats (180-200g) were provided by Vital River (Beijing, China). After adapting to the environment for 5 days, rats were randomly divided into two groups. Monocrotaline induced PAH (MCT-PAH) group $(n=10)$ : rats received a single injection of MCT (60mg kg-1, Sigma, USA) subcutaneously. Control group $(n=10)$ : rats received a single subcutaneous injection of $0.9 \%$ saline at the same volume as the MCT injection. Additionally, dynamic Cyr61 expression in lung tissue at different time points (1, 2, 3 and 4 weeks) were analyzed (3 rats for each group at each time point). All procedures were performed according to the protocols approved by Animal Research Ethics Committee of Peking University Health Science Center (permit number J201527) and the guide for Care and use of laboratory animals published by the US National Institutes of Health (NIH Publication Number 85-23, revised 1996).

\section{Hemodynamic analysis}

After 21 days, rats were anaesthetized with pentobarbital sodium via intraperitoneal injection (60 $\mathrm{mg}$ kg-1, Sigma, USA). After a tracheotomy, the right external jugular vein was separated and a polyethylene 50 catheter connected to a pressure transducer was inserted into the right external jugular vein. Then polyethylene 50 catheter was threaded into the right ventricle and pulmonary artery to measure right ventricular systolic pressure (RVSP) and mean pulmonary arterial pressure (mPAP) by a polygraph system (Power Lab 8/30; AD Instruments, Sydney, Australia). Right ventricular hypertrophy evaluation has been described in our previous study $[13,14]$. Rats were eventually sacrificed, the left lung tissues and the upper left pulmonary arteries were harvested and stored at $-80^{\circ} \mathrm{C}$ until use, and the remnant lung tissues were fixed by formalin until use.

\section{Cell isolation, culture and identification}

PASMCs were obtained from rat intrapulmonary arteries (third or fourth division) as previously described $[15,16]$. Briefly, male Sprague-Dawley rats (3 weeks after vehicle or MCT injection) from control group and MCT-induced PAH group were anesthetized with $130 \mathrm{mg}$ kg-1 pentobarbital sodium intraperitoneally. The hearts and lungs were then removed and transferred to a dish full of cold physiological salt solution containing $130 \mathrm{mM} \mathrm{NaCl}, 5$ $\mathrm{mM} \mathrm{KCl}, 1.2 \mathrm{mM} \mathrm{MgCl}$, $1.5 \mathrm{mM} \mathrm{CaCl} 2,10 \mathrm{mM}$ HEPES and $10 \mathrm{mM}$ glucose, with $\mathrm{pH}$ adjusted to 7.4 with $5 \mathrm{M} \mathrm{NaOH}$. Fixed the tissue and exposed the intrapulmonary arteries, afterwards isolated and cleaned connective tissue and adventitia. The endothelium was removed by rubbing the luminal surface with a cotton swab. The arteries were transferred to $\mathrm{cool}\left(4^{\circ} \mathrm{C}\right)$ physiological salt solution to recover for $30 \mathrm{~min}$. Next, the tissues were digested at $37^{\circ} \mathrm{C}$ for $20 \mathrm{~min}$ in reduced-Ca2+ physiological salt solution containing $1,750 \mathrm{U} / \mathrm{ml}$ typeIcollagenase, $9.5 \mathrm{U} / \mathrm{ml}$ papain, $2 \mathrm{mg} / \mathrm{ml}$ bovine serum albumin and $1 \mathrm{mM}$ dithiothreitol. After centrifuged and resuspended, the cells were transferred to $6-\mathrm{cm}$ petri dishes in DMEM (containing $5.5 \mathrm{mM}$ glucose, Gibco, C11995500BT, 4.5g/1 D-Glucose) containing 10\% fetal bovine serum, and incubated in a humidified atmosphere containing $5 \% \mathrm{CO} 2$ in air at $37^{\circ} \mathrm{C}$. PASMCs identity was verified by a-actin immunofluorescent staining. Protein was extracted and the levels of calponin-1 and a-actin were detected by Western blot as described below.

\section{Immunohistochemical analysis}

After hemodynamic measurement, all of the rats were sacrificed and the lung tissues were prepared for immunohistochemical analysis. Briefly, removed the lung tissues from formalin, embedded them in paraffin, and sectioned the paraffin blocks in 5-um thicknesses. After deparaffinized, the tissues were stained with primary anti-Cyr61 antibody (Abcam, USA) in a 1:250 dilution overnight at $4^{\circ} \mathrm{C}$, and then with horseradish peroxidase-conjugated secondary antibody for 1 hour at room temperature. For color reactions, diaminobenzidine was used and counter stained with hematoxylin. The staining was analyzed by using Leica microscope and Image Pro-Plus image software.

\section{Western blot analysis}

The homogenate of lung tissues, pulmonary arteries, and PASMCs were prepared and subjected to electrophoresis on sodium-dodecyl-sulfate polyacrylamide gels, and then transferred onto polyvinylidene fluoride membranes. Membranes were blocked with $5 \%$ skimmed milk or $5 \%$ bovine serum albumin, and incubated with monoclonal antibody including anti-Cyr61 (Abcam, USA), anti-AKT (Cell signaling Technology, USA), anti-p-AKT (Cell signaling Technology, USA), anti $\beta$-actin (Sigma-Aldrich, St Louis, MO, USA) or anti-GAPDH (ZSGB-BIO, China) at $4^{\circ} \mathrm{C}$ overnight, followed by the matched secondary antibody (Protein Tech, Chicago, IL, USA). Immunopositive spots were probed using ECL-PlusTM (Amersham Biosciences, Chalfont St Giles, UK).

\section{Quantitative real-time polymerase chain reaction (qRT-PCR)}

Total RNA was extracted from the lung tissues and pulmonary arteries with Trizol (Life Technologies, USA) according to the standard 
protocol. cDNA was generated from 2 ug total RNA using High capacity cDNA Reverse Transcription Kit (Applied Biosystems, USA). Two-step real-time PCR was performed using SYBR Green Realtime PCR Master Mix (Applied Biosystems, USA) according to the manufacturer's instructions in 7500 Real Time PCR System (Applied Biosystems, USA). Thermocycler conditions were shown as follows: initial holding at $95^{\circ} \mathrm{C}$ for 60 seconds followed by a 2-step PCR process that consisted of $95^{\circ} \mathrm{C}$ for 15 seconds and $60^{\circ} \mathrm{C}$ for 60 seconds for 40 cycles. Primer sequences of Cyr61 were 5'-CTCAACGAGGACTGC

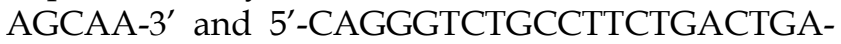
3'. ABI Prism 7500 sequence detection system (Applied Biosystems, USA) was used for quantitative analysis. The level of target gene expression was normalized against the $\beta$-actin gene by the difference in cycle threshold $(\Delta \mathrm{Ct})$. Then the difference between the $\Delta \mathrm{Ct}$ values of the test samples and those of the controls was expressed as $\Delta \Delta \mathrm{Ct}$. Relative expression of Cyr61 was calculated as $2^{-\Delta \Delta} \mathrm{Ct}$.

\section{In Vitro cell proliferation assay}

PASMCs from PAH rats were seeded at $3 \times 10^{3}$ cells per well in 96-well plates overnight, after placing in serum-starved condition for a further $24 \mathrm{~h}$, various concentrations $(0,200,2000 \mathrm{ng} / \mathrm{ml})$ of exogenous recombinant Cyr61 protein (PeproTech, USA) were added and incubated for 72 hours. Cell Counting Kit-8 kit (CCK8) was used to detect the proliferation of cells. Briefly, 10 ul of CCK8 (Dojindo, Japan) solution was added into the wells and incubated for an additional 2 hours, then the optical density for each well was measured at a wavelength of $450 \mathrm{~nm}$.

\section{RNAi knockdown of Cyr61 gene expression}

Cyr61 small interfering RNA (siCyr61) was designed and synthesized by Life Technology (USA). PASMCs from normal rats were cultured in 6-well plates. A transfection mixture of siCyr61 oligonucleotides and lipofectamine RNAIMix (life technologies, USA) in serum-free medium was added to PASMCs according to the manufacturer's instructions. After 6 hours, the medium was replaced with complete DMEM containing 10\% FBS for an additional 48 hours. At the end of culture, expression of Cyr61 in PASMCs was analyzed using real-time PCR and western blot as described above; AKT and p-AKT were detected using western blot. In addition, cell proliferation in PASMCs was measured as described above.

\section{Statistical analysis}

All experiment data were from at least three separate experiments, and performed by $t$-text or one-way analysis of variance using SPSS version 18.0 (SPSS, USA). Summary statistics were expressed as means \pm standard deviation (SD). A P value of $<0.05$ was considered as being statistically significant.

\section{Results}

\section{Plasma Cyr61 levels in patients and healthy controls}

The baseline characteristics of patients and healthy control participants were shown in Table 1. Their age and sex were comparable. ELISA assay showed plasma Cyr61 concentrations in PAH patients were notably elevated in CTD-PAH and IPAH patients $(229.36 \pm 123.40 \mathrm{pg} / \mathrm{ml})$ compared with CTD without PAH patients $(174.09 \pm 78.43 \mathrm{pg} / \mathrm{ml})$ and the healthy volunteers $(111.46 \pm 21.68 \mathrm{pg} / \mathrm{ml})$. In particularly, CTD-PAH patients had the highest Cyr61 concentration $(295.00 \pm 147.33 \mathrm{pg} / \mathrm{ml})$ among the four groups. Meanwhile, the level of Cyr61 in IPAH was statistically higher than in healthy controls $(P<0.001)$, and a trend to be higher than that of CTD without $\mathrm{PAH}$ patients, but the difference was not statistically significant $(P>0.05)$. (Fig. 1).

Table 1. Baseline characteristics of patients and controls

\begin{tabular}{lllll}
\hline & $\begin{array}{l}\text { Healthy } \\
\text { Controls } \\
(\mathrm{n}=28)\end{array}$ & $\begin{array}{l}\text { CTD without } \\
\text { PAH } \\
(\mathrm{n}=29)\end{array}$ & $\begin{array}{l}\text { CTD-PAH } \\
(\mathrm{n}=20)\end{array}$ & $\begin{array}{l}\text { IPAH } \\
(\mathrm{n}=20)\end{array}$ \\
\hline $\begin{array}{l}\text { Age (years, } \\
\text { mean } \pm \text { SD })\end{array}$ & $42.45 \pm 16.19$ & $41.83 \pm 17.09$ & $40.07 \pm 14.90$ & $41.65 \pm 15.72$ \\
Sex (female/male) & $25 / 3$ & $25 / 4$ & $18 / 2$ & $18 / 2$ \\
\hline
\end{tabular}

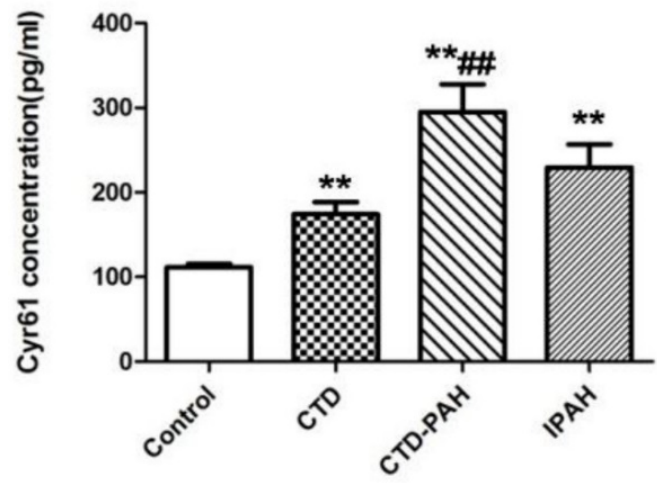

Fig 1. Concentration of Cyr61 in plasma. Cyr61 concentration in plasma from healthy controls $(n=28)$, CTD patients without PAH $(n=29)$, CTD-PAH patients $(n=20)$ and IPAH patients $(n=20)$ were determiend by ELISA. $* * P<0.001$ vs. controls, \# $\mathrm{P}<0.001$ vs. CTD patients.

\section{Hemodynamic evaluation of rats}

The MCT-induced rats had a significantly higher mPAP and RVSP compared with the control rats (36.69 $\pm 3.24 \mathrm{mmHg}$ vs. $18.51 \pm 0.04 \mathrm{mmHg}, P=0.001$; and 

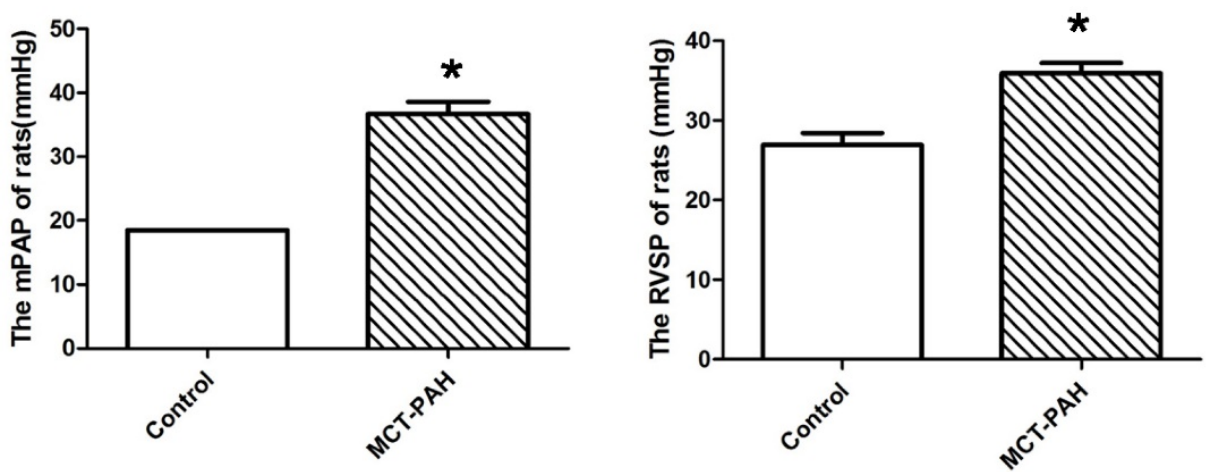

Fig 2. Pulmonary haemodynamic parameters of control rats and the MCT-induced rats. $(A)$, mean pulmonary arterial pressure (mPAP). (B), right ventricular systolic pressure (RVSP). $* \mathrm{P}<0.05$ vs. control.
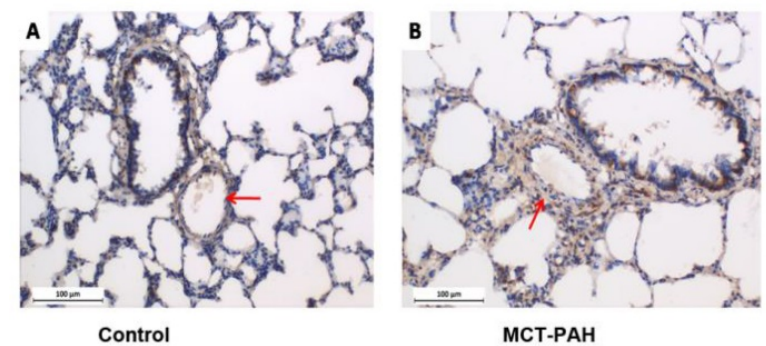

МСТ-РАH

Fig 3. Expression of Cyr61 in lung tissues. Immunohistochemistry was used to determine the expression of Cyr61 in lung tissues of control rats $(\mathrm{A})$ and MCT-PAH rats $(\mathrm{B})$. ${ }^{*} \mathrm{P}<0.05 \mathrm{MCT}-\mathrm{PAH}$ rats vs. control rats.

A

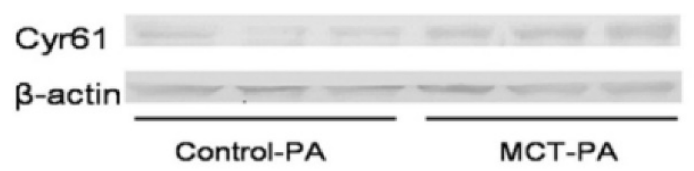

Control-PA

MCT-PA

B

Cyr61

$\beta$-actin

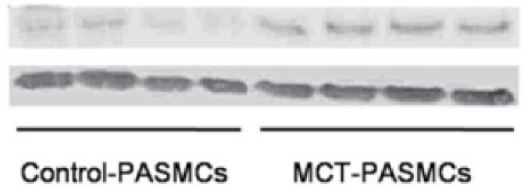

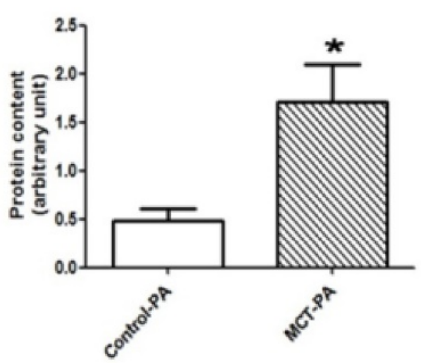

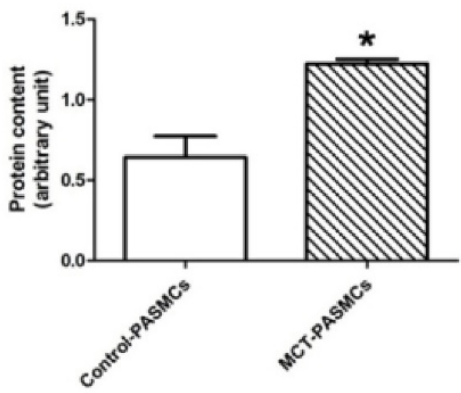

Fig 4. Expressions of Cyr61 in pulmonary arteries and pulmonary arterial smooth muscle cells (PASMCs). Expressions of Cyr61 inpulmonary arteries (A) and PASMCs (B) from control rats and MCT-treated rats were detected by western-blot. $* \mathrm{P}<0.05 \mathrm{MCT}-\mathrm{PAH}$ rats vs. control rats.

$35.97 \pm 2.80 \mathrm{mmHg} \quad$ vs. $\quad 26.91 \pm 3.42 \mathrm{mmHg}, \quad P=0.002$ respectively) (Fig. 2), which represented the MCT induced PAH rat model was established successfully.

\section{The expression of Cyr61 in lung tissues and pulmonary arteries}

Expression of Cyr61 in lung tissues was first investigated by morphologic analysis. By immunohis-

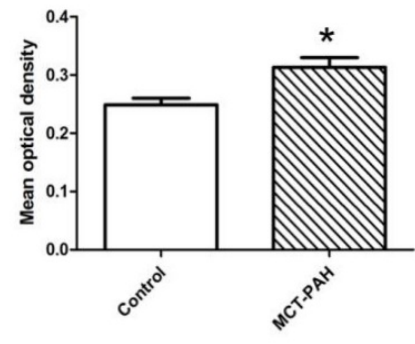

tochemical staining, we found that Cyr61 was extensively expressed in lung tissues, especially in pulmonary arteries $(70-500 \mu \mathrm{m}$ in diameter). Higher expression of Cyr61 was observed in the pulmonary arteries from MCTinduced PAH rats than that of control rats when analyzed by Image Pro-Plus image software (Fig. 3). Furthermore, both the levels of Cyr61 protein and mRNA (Fig. 4A) in pulmonary arteries were higher in MCT-induced PAH rats compared with controls. Additionally, the investigation of dynamic Cyr61 expression in lung tissue showed that Cyr61 was highly expressed from the early to advanced stage of PAH (the 1st week after MCT injection to the 3rd week). Interestingly, it seems that the expression of Cyr61 was decreased over time in the MCT-induced PAH rats, and no statistical significance was observed at the 4 th week after MCT injection between MCT-induced $\mathrm{PAH}$ rats and control rats (Fig.5).

\section{The identification of PASMCs}

After a high purity of PASMCs was identified by a-actin staining (Fig. 6), the expression of calponin-1 and a-actin in PASMCs from MCT-treated rats were found to be significantly decreased than the control rats (Fig. 7), which indicated that the PASMCs from MCT-treated rats underwent a transformation to a proliferative phenotype (in synthetic state rather than contractile state).

\section{The expression of Cyr61 in PASMCs}

A significantly elevated expression of Cyr61 in PASMCs from MCT-treated rats compared with the control rats was shown by western blot (Fig. 4B). 


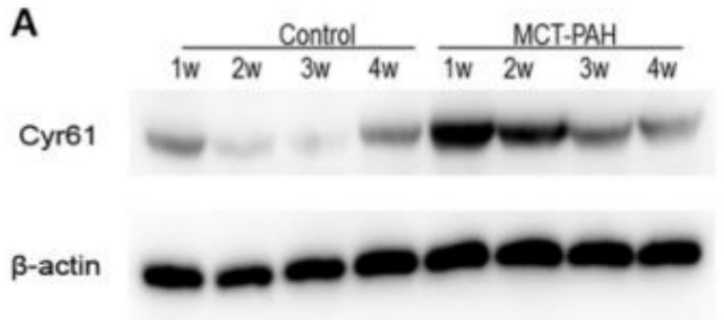

B

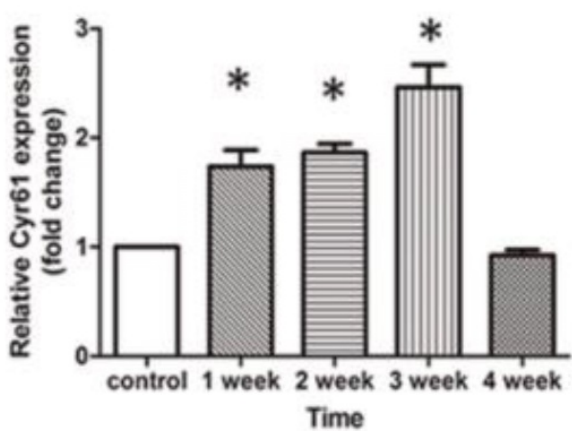

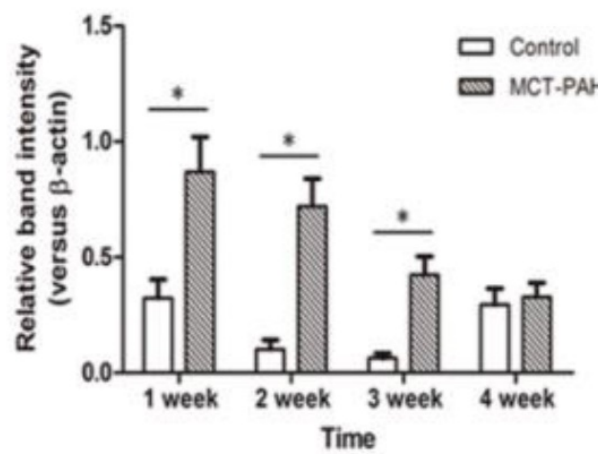

Fig 5. Expression of Cyr61 in lung tissues. The levels of Cyr61 protein (A) and mRNA (B) in lung tissues of MCT-induced PAH rats and control rats were detected by western-blot and real-time PCR respectively. ${ }^{*} \mathrm{P}<0.05 \mathrm{MCT}-\mathrm{PAH}$ rats vs. control rats.

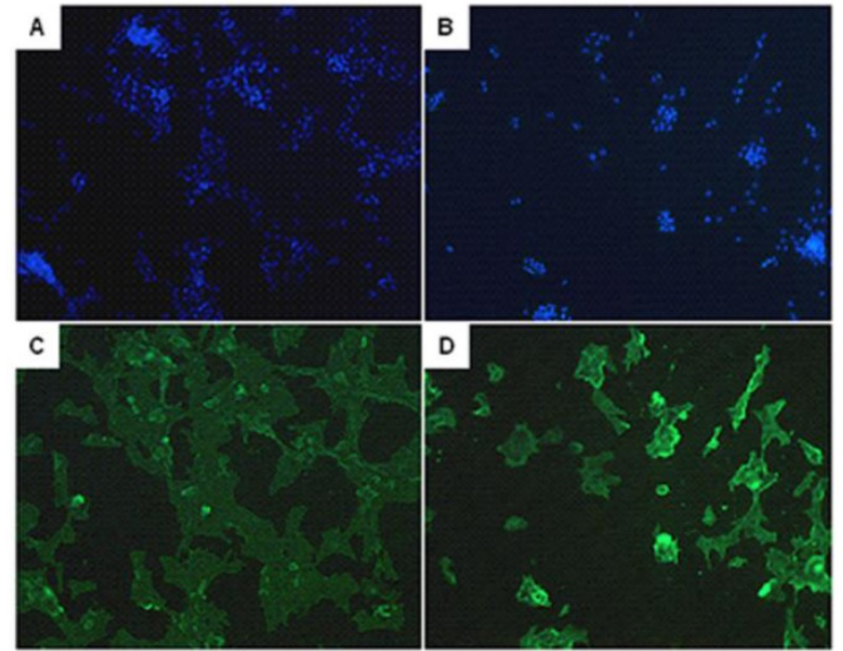

Fig 6. The identification of pulmonary arterial smooth muscle cells (PASMCs). Immunofluorescent staining of the DAPI (A, B) and $\alpha$-actin (C, D) in PASMCs. A and $C$ represent PASMCs from control rats. $B$ and $D$ represent PASMCs from MCT induced $\mathrm{PAH}$ rats.

\section{Exogenous recombinant Cyr61 protein promoted the proliferation of PASMCs}

After the treatment of PASMCs with different concentration of exogenous recombinant Cyr61 protein $(0,200,2000 \mathrm{ng} / \mathrm{ml})$, the proliferation of PASMCs were tested by CCK8 experiment. The data showed that Cyr61 protein promoted the proliferation of PASMCs in a dose-dependent manner (Fig. 8).

\section{Critical role of p-AKT in Cyr61 mediated PASMCs proliferation}

The specific siRNA for Cyr61 which is able to reduce expression of both Cyr61 mRNA and protein by over $60 \%$ was verified (Fig. 9A). The following CCK8 assay showed that the proliferation of PASMCs was reduced when the expression of Cyr61 was inhibited by siRNA, which further confirmed that Cyr61 played a role in enhancing the PASMCs proliferation (Fig. 9B). The result further showed that the expression of p-AKT was decreased significantly after the expression of Cyr61 was inhibited, while the expression of total AKT did not change (Fig. 9C).

\section{Discussion}

With the impressive progression in the pathogenesis of $\mathrm{PAH}$ during the past decades, $\mathrm{PAH}$ has been regarded as a vasculopathy where excessive migration and proliferation of pulmonary vascular cells from intima, media and adventitia membranes in combination with dysregulated immune responses lead to inappropriate pulmonary vascular remodeling $[3,17]$. Despite the understanding of the pathogenesis and the therapeutic options for $\mathrm{PAH}$ have been improved, the survival of $\mathrm{PAH}$ patients remains poor [18]. Thus, a better understanding and discovery of novel pathways and therapeutic targets in $\mathrm{PAH}$ pathogenesis may contribute to the further improvement in the diagnosis and treatment of this devastating disease. 

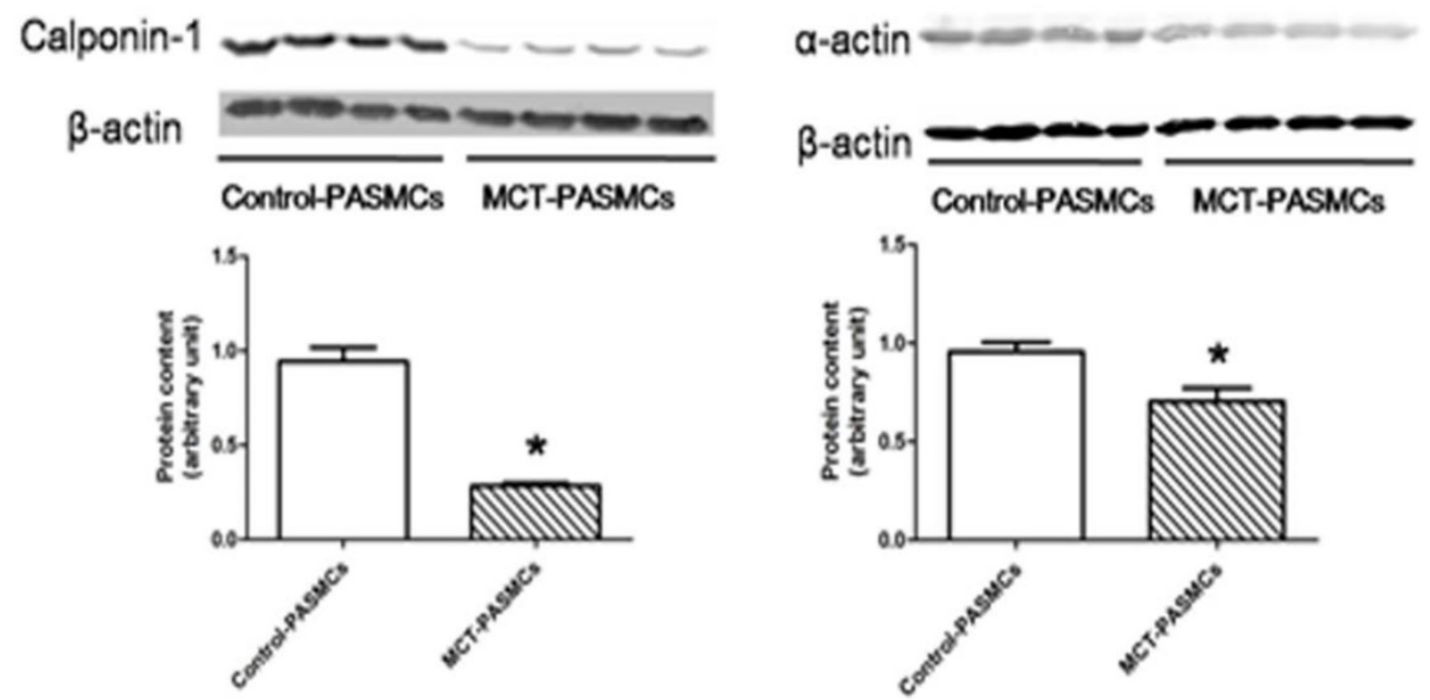

Fig 7. Expressions of calponin- 1 and $\alpha$-actin in pulmonary arterial smooth muscle cells (PASMCs). Expressions of calponin-1 and $\alpha$-actin in PASMCs from control rats and MCT-treated rats were determined by western-blot. $* \mathrm{P}<0.05 \mathrm{MCT}-\mathrm{PAH}$ rats vs. control rats.

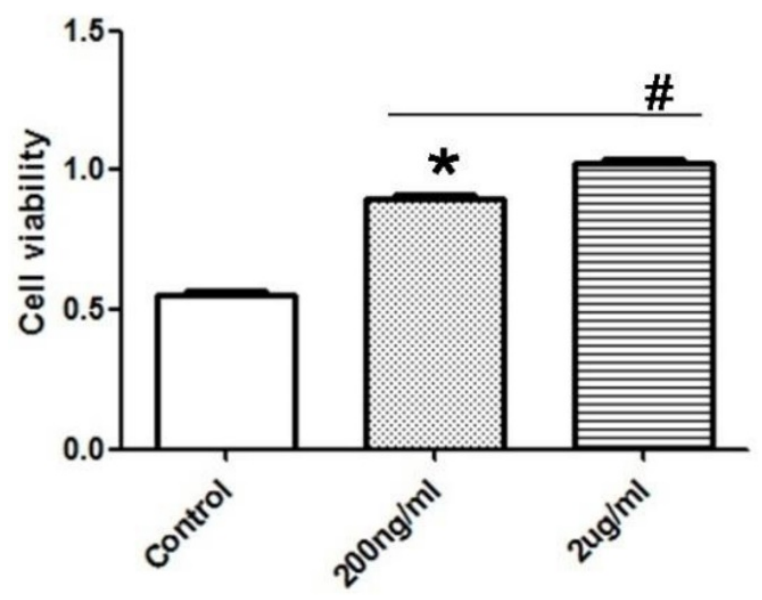

Fig 8. Exogenous recombinant Cyr61 protein promotes the proliferation of PASMCs. PASMCs were treated with exogenous recombinant Cyr61 protein $(0,200$, $2000 \mathrm{ng} / \mathrm{ml})$, then the proliferation was assessed by CCK8 kit. $* \mathrm{P}<0.05$ MCT-PAH rats vs. control rats, \# P<0.05 $2 \mathrm{ug} / \mathrm{ml}$ vs. $200 \mathrm{ng} / \mathrm{ml}$.

Cyr61 was the first cloned classical member of the CCN family, a series of matricellular proteins responsible for many physiological and pathological processes. Previous studies have shown that Cyr61 regulates not only angiogenesis, chondrogenesis, and wound healing, but also cell proliferation, adhesion, migration $[19,20]$. Currently, Cyr61 is considered as a novel inflammatory modulator, which has been implicated in leukocyte migration and inflammatory process [10]. For instance, after investigation in rheumatoid arthritis (RA) for many years, overexpressed Cyr61 has been found in synovial tissue, fibroblast-like synoviocyte and synovial fluid. Elevated Cyr61 in RA synovial fluid could promote the proliferation of fibroblast-like synoviocytes and neutrophil infiltration [21, 22]. In collagen-induced arthritis animal model, anti-Cyr61 monoclonal antibody could attenuate inflammation and retard disease progression [23]. Cyr61 was also shown being involved in the development of vascular diseases. However, the role of Cyr61 in PAH has not been explored clearly.

Our study showed that the level of Cyr61 in the plasma of PAH patients was significantly higher than that of healthy individuals, suggesting that Cyr61 may participate in the pathogenesis of PAH. In patients with CTD-PAH, the Cyr61 concentration in plasma was statistically higher than that of CTD without PAH patients, and a trend of increase compared to that of IPAH patients, further indicating the potential influence of Cyr61 on PAH. Of course, there are some shortcomings of this study, for instance, the small sample size and heterogeneity of underlying connective tissue diseases. To solve these problems, we have initiated a separate national multiple center study to look at the expression of plasma Cyr61 in patients with systemic lupus erythematosus associated PAH (SLE-PAH), which counts for almost half of all CTD-PAH in China [24].

With the aid of animal experiments, we noted that Cyr61 was extensively expressed in lung tissues, but mainly located in pulmonary arteries. Although PASMCs were previously reported as the major cells generating Cyr61, Cyr61 actually could also be produced by various cells in lung tissues, such as alveolar epithelial cells, fibroblasts, pulmonary artery endothelial cells and immune cells. Cyr61 functions in a paracrine/autocrine manner, therefore lung tissue derived Cyr61 may have biological influence on adjacent pulmonary vascular cells. By investigating the dynamic expression of Cyr61 in lung tissue at 
different time points $(1,2,3,4$ weeks after MCT injection), we found that Cyr61 was highly expressed from the early to the advanced stage of $\mathrm{PAH}$ development compared with the control rats. Interestingly, a trend of decreasing Cyr61 expression was observed over time. We speculated that Cyr61, as a new pro-inflammatory mediator, could stimulate the production of some pro-inflammatory cytokines (such as IL-1 $\beta$ and IL-6) and promote Th17 differentiation [23, 25]. Meanwhile, secretion of Cyr61 could be induced by pro-inflammatory stimuli which generate a positive feedback loop. For the inflammatory mechanism in $\mathrm{PAH}$ pathogenesis, the initial hit (such as genetic susceptibility, infections, drugs, and cytokines) results in an acute injury and dysfunction of pulmonary vascular endothelial cells and subsequently massive pro-inflammatory cytokines and chemokines are released. These pro-inflammatory cytokines and chemokines then may drive vascular remodeling processes directly or via bypassing signaling pathways [26]. Therefore, it is possible that Cyr61 is expressed higher at the early stage of PAH development than that at the advanced stage when tissue repairing and vascular remodeling occur. Our speculations need to be proved by further studies.

We also revealed higher expression of Cyr61 in pulmonary arteries and PASMCs of MCT-induced $\mathrm{PAH}$ rats than the control rats, indicating Cyr61 was indeed highly expressed in the PASMCs of MCT-treated PAH rats. Furthermore, vitro studies

A

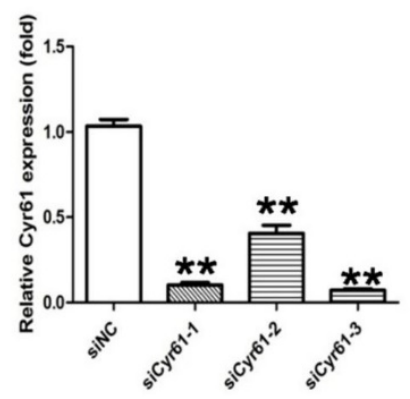

B

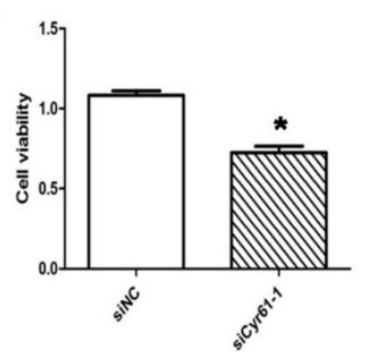

C
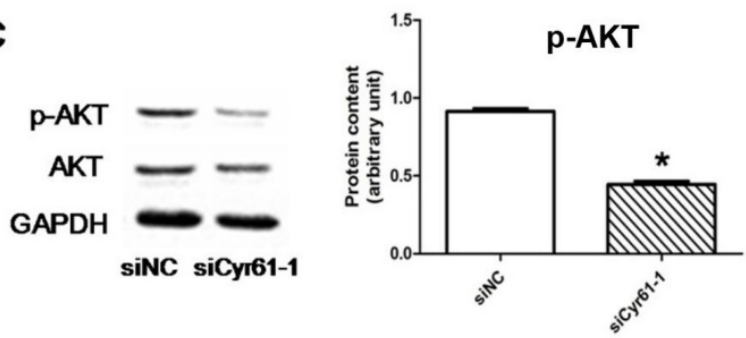

Fig 9. Signaling pathways involved in Cyr61 mediated PASMCs proliferation. (A), 3 identified specific siRNA for Cyr61 (siCyr61-1, siCyr61-2, siCyr61-3) that could reduce expression of both Cyr61 mRNA and protein. siCyr61-1 worked the best. (B), CCK8 assay showed that the proliferation of PASMCs was reduced when the expression of Cyr61 was inhibited. (C), Western-blot showed that expression of p-AKT was decreased significantly, while the expression of total AKT did not change in PASMCs when the expression ofCyr61 was inhibited. $* \mathrm{P}<0.05$ siCyr61-1 vs. siNC, $* * \mathrm{P}<0.05$ siCyr61-1/2/3 vs siNC. showed that exogenous recombinant Cyr61 protein promoted the proliferation of PASMCs. When the expression of Cyr61 was down-regulated, the proliferation of PASMCs was reduced significantly. Importantly, our data is consistent with a previous report showing knockdown of Cyr61 substantially inhibited the proliferation of aorta vascular smooth muscle cells and suppressed the neointimal hyperplasia in a rat carotid artery balloon injury model [27]. Based on the previous study which confirmed that PI3K-AKT was a signal pathway of Cyr61 and functioned as promoting cell survival, we tested the expression of AKT and p-AKT in our study. The results showed that the level of p-AKT was significantly down-regulated, while the expression of total AKT did not change when Cyr61 was inhibited by siRNA in PASMCs, suggesting that PASMCs proliferation could be regulated by Cyr61 through the activation of AKT. Taken together, the over-expression of Cyr61 in PAH patients and MCT-induced PAH rats in combination with the functional evidence of promoting PASMCs proliferation supports that Cyr61 may play an important role in the pathogenesis of $\mathrm{PAH}$.

Coincidently, almost at the same time, American researchers found the similar result of upregulation of Cyr61 expression in hypoxia induced pulmonary hypertension mice. However, they noticed bioactive recombinant Cyr61 significantly suppressed hypoxiainduced contraction in human PASMCs in vitro and a protection role of Cyr61 in hypoxia induced pulmonary hypertension [11]. The difference may be explained by that Cyr61 may function in different ways through distinct receptors including integrins and heparin sulfate proteoglycans in a cell-type and context-dependent manner [20]. For instance, Cyr61 is known as a double-edged sword in cancer for its ability in either promoting cell proliferation, survival and angiogenesis by binding to integrin $\operatorname{av} \beta 3$ or inducing apoptosis and cellular senescence through integrin a6ß1 and heparin sulfate proteoglycans [20, 28]. There are also differences between hypoxia induced $\mathrm{PH}$ model and MCT-induced PAH model, which represent different types 
of $\mathrm{PH}$ and associate with different diseases $[29,30]$.

Due to the unavailability of ELISA kit for rat, we are not able to detect the level of Cyr61 in the plasma of our animal models. Similarly, due to the unavailability of anti-Cyr61 neutralizing monoclonal antibody and probably the lethality if Cyr61 was knocked out, we could not directly block or knock out the action of Cyr61 in PAH rats. And we will be committed to this area in the future.

In summary, we revealed increased Cyr61 levels in plasma from patients with IPAH and CTD-PAH. Meanwhile, upregulated expression of Cyr61 was observed in lung tissues, pulmonary arteries and PASMCs from MCT induced PAH rat models. Further study indicated that Cyr61 could promote the proliferation of PASMCs in PAH rats via AKT pathway. Recently, perivascular inflammation during the process of vascular remodeling of PAH has drawn increasing attention. We speculate Cyr61 contributes to the pathogenesis of PAH just by involving inflammation and proliferation. Our study of Cyr61 on the pathogenesis of PAH via inflammatory pathway is ongoing. A better understanding of Cyr61 in the pathogenesis of PAH may contribute to identify the potential therapeutic target and to improve the prognosis.

\section{Acknowledgements}

This work was supported by National Natural Science Foundation of China (No: 91442120).

\section{Author Contribution}

Zhang and Jing designed the study; Gao, Fan, Hao, Yuan and Liu did the bench work and prepared all figures and tables; Fan, Gao, Hao, Jing, Zhang prepared the manuscript. All authors reviewed the manuscript. Final approval of the version: Zhuoli Zhang.

\section{Competing Interests}

None.

\section{References}

1. Simonneau G, Gatzoulis MA, Adatia I, Celermajer D, Denton C, Ghofrani A, et al. Updated clinical classification of pulmonary hypertension. Journal of the American College of Cardiology. 2013; 62: D34-41.

2. Galie N, Humbert M, Vachiery JL, Gibbs S, Lang I, Torbicki A, et al. 2015 ESC/ERS Guidelines for the diagnosis and treatment of pulmonary hypertension: The Joint Task Force for the Diagnosis and Treatment of Pulmonary Hypertension of the European Society of Cardiology (ESC) and the European Respiratory Society (ERS): Endorsed by: Association for European Paediatric and Congenital Cardiology (AEPC), International Society for Heart and Lung Transplantation (ISHLT). Eur Respir J. 2015; 46: 903-75.

3. Schermuly RT, Ghofrani HA, Grimminger MRWA. Mechanisms of disease: pulmonary arterial hypertension. Nature Review Cardiology. 2011; 40: 1055-60

4. Gaine S. Pulmonary Hypertension. JAMA. 2000; 284: 3160

5. Rabinovitch M, Guignabert C, Humbert M, Nicolls MR. Inflammation and Immunity in the Pathogenesis of Pulmonary Arterial Hypertension. Circulation Research. 2014; 115: 165-75.

6. Grunig G, Durmus N. Spotlight on Inflammation in Pulmonary Hypertension. Am J Respir Crit Care Med. 2015; 192: 913-5.
7. Mo FE, Muntean AG, Chen CC, Stolz DB, Watkins SC, Lau LF, CYR61 (CCN1) is essential for placental development and vascular integrity. Mol Cell Biol. 2002; 22: 8709-20.

8. Estrada $\mathrm{R}, \mathrm{Li} \mathrm{N}$, Sarojini $\mathrm{H}$, An J, Lee MJ, Wang E. Secretome from mesenchymal stem cells induces angiogenesis via Cyr61. J Cell Physiol. 2009; 219: 563-71.

9. Lee H-Y, Chung J-W, Youn S-W, Kim J-Y, Park K-W, Koo B-K, et al. Forkhead Transcription Factor FOXO3a Is a Negative Regulator of Angiogenic Immediate Early Gene CYR61, Leading to Inhibition of Vascular Smooth Muscle Cell Proliferation and Neointimal Hyperplasia. Circulation Research. 2007; 100: 372-80.

10. Emre Y, Imhof BA. Matricellular protein CCN1/CYR61: a new player in inflammation and leukocyte trafficking. Seminars in immunopathology. 2014; 36: 253-9.

11. Lee SJ, Zhang M, Hu K, Lin L, Zhang D, Jin Y. CCN1 suppresses pulmonary vascular smooth muscle contraction in response to hypoxia. Pulmonary circulation. 2015; 5: 716-22.

12. Hoeper MM, Bogaard HJ, Condliffe R, Frantz R, Khanna D, Kurzyna M, et al. Definitions and diagnosis of pulmonary hypertension. Journal of the American College of Cardiology. 2013; 62: D42-50.

13. Yuan $\mathrm{P}, \mathrm{Wu} W \mathrm{~W}$, Gao $\mathrm{L}$, Zheng $\mathrm{ZQ}$, Liu $\mathrm{D}$, Mei $\mathrm{HY}$, et al. Oestradiol ameliorates monocrotaline pulmonary hypertension via NO, prostacyclin and endothelin-1 pathways. Eur Respir J. 2013; 41: 1116-25.

14. Fan YF, Zhang $R$, Jiang $X$, Wen $L$, Wu DC, Liu $D$, et al. The phosphodiesterase- 5 inhibitor vardenafil reduces oxidative stress while reversing pulmonary arterial hypertension. Cardiovasc Res. 2013; 99: 395-403.

15. Wang J, Shimoda LA, Sylvester JT. Capacitative calcium entry and TRPC channel proteins are expressed in rat distal pulmonary arterial smooth muscle. Am J Physiol Lung Cell Mol Physiol. 2004; 286: L848-58.

16. Wang J, Juhaszova M, Rubin LJ, Yuan XJ. Hypoxia inhibits gene expression of voltage-gated $\mathrm{K}+$ channel alpha subunits in pulmonary artery smooth muscle cells. J Clin Invest. 1997; 100: 2347-53.

17. Guignabert C, Tu L, Girerd B, Ricard N, Huertas A, Montani D, et al. New Molecular Targets of Pulmonary Vascular Remodeling in Pulmonary Arterial Hypertension. CHEST Journal. 2015; 147: 529.

18. Humbert M, Sitbon O, Chaouat A, Bertocchi M, Habib G, Gressin V, et al. Survival in patients with idiopathic, familial, and anorexigen-associated pulmonary arterial hypertension in the modern management era. Circulation. 2010; 122: 156-63.

19. Perbal B. CCN proteins: multifunctional signalling regulators. The Lancet. 2004; 363: 62-4.

20. Lau LF. CCN1/CYR61: the very model of a modern matricellular protein. Cellular and molecular life sciences: CMLS. 2011; 68: 3149-63.

21. Zhu X, Xiao L, Huo R, Zhang J, Lin J, Xie J, et al. Cyr61 is involved in neutrophil infiltration in joints by inducing IL-8 production by fibroblast-like synoviocytes in rheumatoid arthritis. Arthritis Res Ther. 2013; 15: R187.

22. Zhang Q, Wu J, Cao Q, Xiao L, Wang L, He D, et al. A critical role of Cyr61 in interleukin-17-dependent proliferation of fibroblast-like synoviocytes in rheumatoid arthritis. Arthritis Rheum. 2009; 60: 3602-12.

23. Lin J, Zhou Z, Huo R, Xiao L, Ouyang G, Wang L, et al. Cyr61 induces IL-6 production by fibroblast-like synoviocytes promoting Th17 differentiation in rheumatoid arthritis. J Immunol. 2012; 188: 5776-84.

24. Hao YJ, Jiang X, Zhou W, Wang Y, Gao L, Wang Y, et al. Connective tissue disease-associated pulmonary arterial hypertension in Chinese patients. Eur Respir J. 2014; 44: 963-72.

25. Zhu X, Song Y, Huo R, Zhang J, Sun S, He Y, et al. Cyr61 participates in the pathogenesis of rheumatoid arthritis by promoting proIL-1beta production by fibroblast-like synoviocytes through an AKT-dependent NF-kappaB signaling pathway. Clinical immunology. 2015; 157: 187-97.

26. Price LC, Wort SJ, Perros F, Dorfmuller P, Huertas A, Montani D, et al. Inflammation in pulmonary arterial hypertension. Chest. 2012; 141: 210-21.

27. Matsumae $H$, Yoshida $Y$, Ono $K$, Togi $K$, Inoue $K$, Furukawa $Y$, et al. CCN1 knockdown suppresses neointimal hyperplasia in a rat artery balloon injury model. Arterioscler Thromb Vasc Biol. 2008; 28: 1077-83.

28. Cheng TY, Wu MS, Hua KT, Kuo ML, Lin MT. Cyr61/CTGF/Nov family proteins in gastric carcinogenesis. World journal of gastroenterology. 2014; 20: 1694-700

29. Stenmark KR, Meyrick B, Galie N, Mooi WJ, McMurtry IF. Animal models of pulmonary arterial hypertension: the hope for etiological discovery and pharmacological cure. Am J Physiol Lung Cell Mol Physiol. 2009; 297: L1013-32.

30. Maarman G, Lecour S, Butrous G, Thienemann F, Sliwa K. A comprehensive review: the evolution of animal models in pulmonary hypertension research; are we there yet? Pulmonary circulation. 2013; 3: 739-56. 